UCT-TP-215/94

June 1994

\title{
Electromagnetic pion form factor at finite temperature
}

\author{
C. A. Dominguez回 \\ Institute of Theoretical Physics and Astrophysics, University of Cape Town, \\ Rondebosch 7700, South Africa \\ M. Loewe \\ Facultad de Fisica, Pontificia Universidad Católica de Chile, Santiago, Chile \\ and \\ J. S. Rozowsky \\ Department of Physics, University of Cape Town, Rondebosch 7700, South Africa
}

\begin{abstract}
The electromagnetic form factor of the pion in the space-like region, and at finite temperature, $F_{\pi}\left(Q^{2}, T\right)$, is obtained from a QCD Finite Energy Sum Rule. The form factor decreases with increasing $\mathrm{T}$, and vanishes at some critical temperature, where the pion radius diverges. This divergence may be interpreted as a signal for quark deconfinement.
\end{abstract}

\footnotetext{
${ }^{1}$ John Simon Guggenheim Fellow 1994-1995
} 
The possibility that QCD exhibits chiral-symmetry restoration and quark-gluon deconfinement at finite temperature has triggered interest in the thermal behaviour of QCD in general, and of hadronic propagators in particular. In this respect, some time ago a proposal was made to consider the imaginary part of any hadronic propagator as a phenomenological order parameter for the deconfinement phase transition [1]. According to this proposal, with increasing $\mathrm{T}$ one should expect resonances to become broader, and stable-hadron propagators to develop a non-zero imaginary part. This behaviour has been confirmed later for pions, nucleons, and rho-mesons using a variety of theoretical approaches [2]. Independent phenomenological evidence for the deconfinement phase transition in QCD may be obtained e.g. by studying the thermal behaviour of the electromagnetic form factor of the pion, $F_{\pi}$. In this case one would expect the size of the pion to increase with increasing temperature. At the critical temperature the pion radius should presumably diverge, indicating quark-gluon deconfinement. A recent calculation in the framework of the Nambu-Jona Lasinio [3] model supports this scenario.

In this note we determine the temperature dependence of $F_{\pi}$ in the space-like region using a Finite Energy QCD Sum Rule (FESR). The pion form factor at $T=0$ has been extensively studied in the past with FESR, as well as with Laplace transform QCD sum rules [4]. In order to establish some notation, as well as the $T=0$ normalization, we briefly describe the method at $T=0$ before introducing thermal corrections.

The appropriate object to study is the three-point function

$$
\Pi_{\mu \nu \lambda}\left(p, p^{\prime}, q\right)=i^{2} \int d^{4} x d^{4} y e^{i\left(p^{\prime} x-q y\right)}<0\left|T\left(A_{\nu}^{\dagger}(x) V_{\lambda}(y) A_{\mu}(0)\right)\right| 0>,
$$

where $A_{\mu}(x)=\bar{u}(x) \gamma_{\mu} \gamma_{5} d(x)$ is the axial-vector current, $V_{\lambda}$ is the electromagnetic current, and $q=p^{\prime}-p$ the momentum transfer. On general analyticity grounds, the three-point function (1) satisfies the double dispersion relation

$$
\Pi_{\mu \nu \lambda}\left(p^{2}, p^{\prime 2}, Q^{2}\right)=\frac{1}{\pi^{2}} \int_{0}^{\infty} d s \int_{0}^{\infty} d s^{\prime} \frac{\rho_{\mu \nu \lambda}\left(s, s^{\prime}, Q^{2}\right)}{\left(s+p^{2}\right)\left(s^{\prime}+p^{\prime 2}\right)},
$$

defined up to subtractions, which are disposed of by Laplace improving the Hilbert transform, or by considering FESR. The QCD sum rule program at $T=0$ [5] may be phrased as follows. The left hand side of (2) in the Euclidean region can be calculated in QCD through the Operator Product Expansion (OPE), to any desired order in perturbation theory, and parametrizing non-perturbative effects in terms of vacuum expectation values of the same quark and gluon fields entering the QCD Lagrangian. Contact with the hadronic world is made by writing the spectral function appearing in the right hand side 
of (2) in terms of all possible hadronic intermediate states contributing to the three-point function. Normally, a reasonable saturation of the dispersion relation is achieved by the ground state, followed after some threshold energy $s_{0}$ by a hadronic continuum modelled by perturbative QCD.

The correlator (1) involves quite a few structure functions, associated with all the Lorentz structures that can be formed with the available four-momenta. In principle, it should not matter which particular structure one chooses to project the pion form factor. We follow [4] in choosing the combination $P_{\mu} P_{\nu} P_{\lambda}$, where $P=p+p^{\prime}$. In this case, the hadronic spectral function in the chiral-limit reads

$$
\left.\rho\left(s, s^{\prime}, Q^{2}\right)\right|_{H A D}=\frac{1}{2} f_{\pi}^{2} F_{\pi}\left(Q^{2}\right) \delta(s) \delta\left(s^{\prime}\right)+\left.\rho\left(s, s^{\prime}, Q^{2}\right)\right|_{Q C D}\left[1-\theta\left(s_{0}-s-s^{\prime}\right)\right],
$$

where $s_{0}$ signals the onset of the continuum, $f_{\pi} \simeq 93 \mathrm{MeV}$, and

$$
\left.\rho\left(s, s^{\prime}, Q^{2}\right)\right|_{Q C D}=\frac{3}{16 \pi^{2}} \frac{Q^{4}}{\lambda^{7 / 2}}\left[3 \lambda\left(x+Q^{2}\right)\left(x+2 Q^{2}\right)-\lambda^{2}-5 Q^{2}\left(x+Q^{2}\right)^{3}\right],
$$

to one-loop order (and in the chiral-limit), with

$$
\lambda=y^{2}+Q^{2}\left(2 x+Q^{2}\right)
$$

and $x=s+s^{\prime}, y=s-s^{\prime}$. Since we are interested in writing the lowest moment FESR for $F_{\pi}$, i.e.

$$
F_{\pi}\left(Q^{2}\right)=\left.\frac{1}{f_{\pi}^{2}} \int_{0}^{s_{0}} d x \int_{-x}^{x} d y \rho\left(x, y, Q^{2}\right)\right|_{Q C D}
$$

rather than a Laplace transform QCD sum rule, the non-perturbative power corrections entering the OPE are of no concern to us here (they contribute to higher moment FESR). The integration region in (6) has been chosen to be a triangle in the (s,s') plane, with base and height equal to $s_{0}$. Other choices of the integration region, e.g. a square region of side $s_{1} \simeq s_{0} / \sqrt{2}$, give similar results. The solution to the FESR (6) is

$$
F_{\pi}\left(Q^{2}\right)=\frac{1}{16 \pi^{2} f_{\pi}^{2}} \frac{s_{0}}{\left(1+Q^{2} / 2 s_{0}\right)^{2}} .
$$

Although not evident from (7), it is important to realize that this analysis is only valid in the region $Q^{2} \geq 1 \mathrm{GeV}^{2}$, where one expects a reasonable convergence of the OPE. This limitation is of no relevance if one is only interested in the thermal behaviour of the ratio 
$F_{\pi}\left(Q^{2}, T\right) / F_{\pi}\left(Q^{2}, 0\right)$. In any case, as shown in [4], Eq.(7) provides a reasonable fit to the experimental data in the region $Q^{2} \simeq 1-4 \mathrm{GeV}^{2}$, if $s_{0} \simeq 1 \mathrm{GeV}^{2}$.

We now proceed to derive the thermal corrections to (6). The vacuum average in Eq.(1) is to be replaced by the Gibbs average

$$
<<\mathrm{A} \cdot \mathrm{B} \cdots>>\equiv \sum_{n} \exp \left(-E_{n} / T\right)<n|\mathrm{~A} \cdot \mathrm{B} \cdots| n>/ \operatorname{Tr}(\exp (-H / T)),
$$

where $|n\rangle$ is any complete set of eigenstates of the (QCD) Hamiltonian, e.g. hadronic states, quark-gluon basis, etc.. If one is to extend smoothly the $T=0 \mathrm{QCD}$ sum rule program to finite temperature, then the natural choice for $\mid n>$ should be the quark-gluon basis, as first proposed in [6]. In fact, assuming the validity of the OPE at $T \neq 0$ and invoking QCD-hadron duality at $T=0$, raising the temperature by an arbitrary small amount would induce very little change in the hadronic spectrum, and in the expectation values of the QCD operators in the OPE. Hence, it is reasonable to expect that the inter-relationship between QCD and hadronic parameters effected by duality will remain valid. An abrupt dissapearance of this inter-relationship as soon as $T \neq 0$ appears highly unlikely. As to the validity of the OPE at finite temperature, one is faced with the same problem as when $T=0$, i.e. no rigorous proof can be given since one is not able to solve QCD analytically and exactly. Instead, other field theory models which are exactly solvable have been used to argue for the validity of the OPE at $T=0$ [7]. This analysis has been extended recently to $T \neq 0[\mathbb{8}$, and it shows the same level of supportive evidence as in the $T=0$ case.

The QCD program at $T \neq 0$ as outlined above leads to some interesting results. For instance, considering the two-point function associated with the axial-vector current, a lowest moment FESR relates $s_{0}(T)$ to $f_{\pi}(T)$ in such a way that $s_{0}(T)$ vanishes at some temperature $T_{d}$ [9]-[10]. This may be interpreted as the critical temperature for deconfinement. In fact, with increasing $\mathrm{T}$, and as resonances begin to melt, the hadronic spectrum should smooth out and $s_{0}(T)$ should decrease. Close to $T_{d}$ the spectral function is then described almost entirely by the quark-gluon degrees of freedom at all energies. Depending on the expression for $f_{\pi}(T)$ used as input, $T_{d}$ could be smaller or almost the same as the critical temperature for chiral-symmetry restoration, $T_{c}$. For instance, using chiral perturbation theory for $f_{\pi}(T)$ leads to $T_{d}<T_{c}$ [9], but this input is only valid at low temperatures. A more refined analysis gives [10

$$
\sqrt{\frac{s_{0}(T)}{s_{0}(0)}} \simeq \frac{f_{\pi}(T)}{f_{\pi}(0)}
$$

and $T_{d}$ only slightly lower than $T_{c}$ (given the accuracy of the method this difference is of 
not much significance).

We have calculated the spectral function (4) at finite temperature using the Dolan-Jackiw formalism. After substitution in (16), the result can be expressed as

$$
F_{\pi}\left(Q^{2}, T\right)=\left.\frac{1}{f_{\pi}^{2}(T)} \int_{0}^{s_{0}(T)} d x \int_{-x}^{x} d y \rho\left(x, y, Q^{2}\right)\right|_{Q C D} F\left(x, y, Q^{2}, T\right)
$$

with

$$
\begin{gathered}
F\left(x, y, Q^{2}, T\right)=1-n_{1}-n_{2}-n_{3}+n_{1} n_{2}+n_{1} n_{3}+n_{2} n_{3}, \\
n_{1}=n_{2} \equiv n_{F}\left(\mid \frac{1}{2 T} \sqrt{\left.\frac{x+y}{2} \mid\right)}\right. \\
n_{3} \equiv n_{F}\left(\left|\frac{Q^{2}+(x-y) / 2}{2 T \sqrt{\frac{x+y}{2}}}\right|\right),
\end{gathered}
$$

and $n_{F}$ is the Fermi thermal factor. In the equations above we have chosen a frame such that $p_{\mu}=(\omega, \mathbf{0})$, and $p_{\mu}^{\prime}=\left(\omega^{\prime}, \mathbf{p}^{\prime}\right)$, in which case

$$
\omega=\sqrt{\frac{x+y}{2}} \quad, \quad \omega^{\prime}=\frac{x+Q^{2}}{2 \sqrt{\frac{x+y}{2}}} .
$$

We have explicitly checked that the ratio

$$
R(T) \equiv \frac{F_{\pi}\left(Q^{2}, T\right)}{F_{\pi}\left(Q^{2}, 0\right)}
$$

is essentially insensitive to other choices of frames. For instance, one may choose $p_{\mu}=$ $(\omega, \mathbf{p})$, and $p_{\mu}^{\prime}=\left(\omega^{\prime},-\mathbf{p}\right)$, which leads to different arguments in the thermal factors, but roughly the same ratio $R(T)$. The temperature behaviour of the ratio (15) is shown in Fig. 1 for $Q^{2}=1 \mathrm{GeV}^{2}$ (solid curve), and $Q^{2}=3 \mathrm{GeV}^{2}$ (broken curve), where we have used (9) together with the results of [10] for $f_{\pi}(T)$. This result for $R(T)$ is in nice agreement with the expectation that as the temperature increases, $F_{\pi}$ should decrease and eventually vanish at the critical temperature for deconfinement $T_{d}$. 
Although the OPE breaks down at small values of $Q^{2}$, one may still extrapolate the ratio (15) into this region just to study the qualitative temperature behaviour of the rootmean-square radius ratio $<r_{\pi}^{2}>_{T} /<r_{\pi}^{2}>_{0}$. Doing this, we find that this ratio increases monotonically with $\mathrm{T}$, doubling at $T / T_{d} \simeq 0.8$, and diverging at the critical temperature. This divergence of $\left\langle r_{\pi}^{2}\right\rangle_{T}$ may be interpreted as a signal for quark deconfinement. In fact, the behaviour of $\left\langle r_{\pi}^{2}\right\rangle_{T}$ can be traced back to the temperature behaviour of the asymptotic freedom threshold $s_{0}(T)$. As $s_{0}(T)$ decreases with increasing $T$, a signature of quark deconfinement, the root-mean-square radius of the pion increases. This result is in qualitative agreement with that obtained in the framework of the Nambu-Jona Lasinio model [3]

\section{Acknowledgements}

The work of (CAD) has been supported in part by the John Simon Guggenheim Memorial Foundation, and that of (ML) by FONDECYT 0751/92. This work has been performed in the framework of the FRD-CONICYT Scientific Cooperation Program.

\section{References}

[1] C.A. Dominguez, Nucl. Phys. B (Proc. Suppl.) 15 (1990) 225; C.A. Dominguez and M. Loewe, Nucl. Phys. B (Proc. Suppl.) 16 (1990) 403.

[2] J.L. Goity and H. Leutwyler, Phys. Lett. B 228 (1989) 517; H. Leutwyler and A.V. Smilga, Nucl. Phys. B 342 (1990) 302; A. Schenk, Nucl. Phys. B 363 (1991) 97; C.A. Dominguez, M. Loewe, and J.C. Rojas, Z. Phys. C - Particles and Fields - 59 (1993) 63; Phys. Lett. B 320 (1994) 377.

[3] H-J. Schultze, J. Phys. G 20 (1994) 531.

[4] V.A. Nesterenko and A.V. Radyushkin, Phys. Lett. B 115 (1982) 410; B.L. Ioffe and A.V. Smilga, Nucl. Phys. B 216 (1983) 373.

[5] M.A. Shifman, A.I. Vainshtein and V.I. Zakharov, Nucl. Phys. B 147 (1979) 385, 448 ; L.J. Reinders, H. Rubinstein and S. Yazaki, Phys. Rep. C 127 (1987) 1.

[6] A.I. Bochkarev and M.E. Shaposnikov, Nucl. Phys. B 268 (1986) 220.

[7] V.A. Novikov, M.A. Shifman, A.I. Vainshtein and V.I.Zakharov, Phys. Rep. C 116 (1984) 103 ; Nucl. Phys. B 249 (1985) 445. 
[8] C.A. Dominguez and M. Loewe, University of Cape Town Report No. UCT-TP208/94 (1994).

[9] C.A. Dominguez and M. Loewe, Phys. Lett. B 233 (1989) 201.

[10] A. Barducci, R. Casalbuoni, S. de Curtis, R. Gatto, and G. Pettini, Phys. Lett. B 244 (1990) 311.

\section{Figure Captions}

Figure 1: Temperature beahviour of the ratio (15) for $Q^{2}=1 \mathrm{GeV}^{2}$ (solid curve), and $Q^{2}=3 \mathrm{GeV}^{2}$ (broken curve). 
This figure "fig1-1.png" is available in "png" format from: http://arxiv.org/ps/hep-ph/9408204v1 